\title{
The Gut Microbiome as Therapeutic Target in Central Nervous System Diseases: Implications for Stroke
}

\author{
Katarzyna Winek $^{1,2,3} \cdot$ Ulrich Dirnagl ${ }^{1,2,3,4,5,6}$ - Andreas Meisel ${ }^{1,2,3,4}$ \\ Published online: 6 October 2016 \\ (C) The Author(s) 2016. This article is published with open access at Springerlink.com
}

\begin{abstract}
Research on commensal microbiota and its contribution to health and disease is a new and very dynamically developing field of biology and medicine. Recent experimental and clinical investigations underscore the importance of gut microbiota in the pathogenesis and course of stroke. Importantly, microbiota may influence the outcome of cerebral ischemia by modulating central nervous system antigenspecific immune responses. In this review we summarize studies linking gut microbiota with physiological function and disorders of the central nervous system. Based on these insights we speculate about targeting the gut microbiome in order to treat stroke.
\end{abstract}

Key Words Gut microbiota $\cdot$ stroke $\cdot$ brain-gut microbiota axis $\cdot$ central nervous system $\cdot$ therapeutic target

Electronic supplementary material The online version of this article (doi:10.1007/s13311-016-0475-x) contains supplementary material, which is available to authorized users.

Andreas Meisel

andreas.meisel@charite.de

1 Department of Experimental Neurology, Charité Universitätsmedizin Berlin, Berlin, Germany

2 NeuroCure Clinical Research, Charité - Universitätsmedizin Berlin, Berlin, Germany

3 Center for Stroke Research Berlin, Charité - Universitätsmedizin Berlin, Berlin, Germany

4 Department of Neurology, Charité - Universitätsmedizin Berlin, Berlin, Germany

5 German Center for Neurodegeneration Research (DZNE), partner site Berlin, Berlin, Germany

6 German Center for Cardiovascular Research (DZHK), Partner Site Berlin, Berlin, Germany

\section{Introduction}

The way we perceive microorganisms and their role in health and disease has changed substantially over the last few decades. It has long been recognized that human-microbial synergy in the gut promotes digestion and contributes to infections when the host-bacterial interaction is disturbed. Recent discoveries, however, have enabled a deeper understanding of the sophisticated interconnection between commensal microbial populations and the host, demonstrating that microbial communities not only refine host metabolism, but also modulate immunity and even contribute to organ development

The first systematic investigations of intestinal bacteria were carried out in the nineteenth century [1]. When investigating stool samples from healthy individuals Friedrich Escherich cultured and characterized the Bacterium coli commune, today known as Escherichia coli and "the work horse of molecular biology" [2]. Over the following decades more commensal microorganisms were isolated and characterized. But it is only the more recent implementation of advanced high-throughput genetic profiling that has given us detailed insight into the cosmos of intestinal microorganisms. Importantly, gut bacteria create a broad-ranging, dynamic microbial community that contributes substantially to processes in the host organism and reacts to changes in host physiology. Joshua Lederberg suggested the term "microbiota" to describe this complex microbial population and defined it as "the ecological community of commensal, symbiotic and pathogenic microorganisms that literally share our body space" [3]. The microbiome is the collective genome of these symbiotic microorganisms and has a cumulative number of genes larger than that in the human genome [4]. Commensal microorganisms contribute substantially to host metabolism, providing vitamins, extracting nutritional components, and metabolizing 
xenobiotics. They fortify the intestinal barrier and provide colonization resistance through passive and active competition with potential pathogens [5]. In recent years, important and previously unanticipated functions of commensal microbiota have been described. The gut microbiota has been shown to contribute to the development of the immune system, and, remarkably, also to the development of the central nervous system (CNS) [6].

Thus, it is not at all a surprising suggestion that gut microbiota might play an important role under pathobiological conditions and in physiological processes. Researchers have identified alterations in the composition of gut microbiota in several diseases, such as inflammatory bowel disease [7], diabetes [8], cancer [9], and disorders of the nervous system, for example pain syndromes [10], Parkinson's disease (PD) [11], spinal cord injury [12], autism [13], and stroke [14-16]. Interestingly, aging is not only a risk factor of stroke, but also affects the gut microbiome [17]. While gut microbiota of older individuals seems to be relatively stable over time, its composition differs substantially from that of younger people and is very heterogeneous [18]. Compelling evidence comes from experimental and clinical studies, and the number of investigations into the role of commensal microbiota in neurological disorders is growing constantly, putting the microbiome to the spotlight in neuroscience. However, the variety and diversity of the diseases, in which the involvement of microbiota has been postulated is tremendous, and only detailed investigations in the coming years will show where the suggested contribution of commensals is legitimate.

Despite all the uncertainties about the role of gut microbiome, commensal microbiota is regarded as a promising therapeutic target and several research groups, start-ups, and large companies have already commenced work on therapies based on the gut microbiome [19, 20]. However, the links between changes in composition of microbiota - which are often the first observation reported in a particular condition - and the course of disease, have to be confirmed as causing or contributing to this condition. This would be the prerequisite for developing microbiome-targeted therapies. In this review we want to discuss evidence for the involvement of gut microbiome in the pathophysiology of CNS disorders and speculate about therapeutic opportunities for manipulations of gut microbiota in CNS disease, with a focus on stroke.

\section{Microbiota and the CNS: Evidence for a Link}

\section{Impact of Gut Microbiota on the "Health" of the CNS: Experimental Studies}

Most insights into the role of microbiota in CNS development and disease originate from studies in germ-free (GF) animals. GF animals have no contact with any microorganisms at any time in their life and are raised in sterile isolators. Establishing the GF animals has proven that life without microbiota is possible [21]; GF organisms, however, have to be provided with exogenous vitamins, otherwise they suffer from severe complications such as vitamin $\mathrm{K}$ deficiency, which leads to improper coagulation [21]. Studies in GF mice have revealed that the GF state leads to alterations in the nervous system (Table 1, for a detailed review see [21]). The enteric nervous system in the absence of microorganisms is not fully developed, and GF mice have an altered intestinal motility [22]. The blood-brain barrier in GF mice is more permeable [23], and microglia from GF mice differ in morphology and function from that of conventionally colonized animals [24]. Myelin component genes in prefrontal cortex of GF animals are upregulated, an observation that correlates with an increased thickness of the myelin sheath in axons of GF mice [25]. Remarkably, GF status influences the behavioral phenotype. Compared with specific pathogen-free (SPF) animals, GF animals have increased motor activity and decreased anxiety accompanied by differentially expressed genes involved in synaptic circuitry [26]. Early colonization of GF animals with conventional microbiota reverses this phenotype [26]. GF mice display an exaggerated stress response due to hyper-responsiveness of the hypothalamus-pituitary-adrenal axis [27], and have impaired memory function [28]. Behavioral phenotypes characteristic for particular strains of rodents (e.g., calm, tranquil nature of BALBc mice) can be transmitted upon transfer of microbiota in experiments using GF and conventionally colonized animals [29]. Neurobehavioral changes attributed to obesity, such as depression and dementia, were transferred with gut microbiome in an experimental mouse model [30]. Several studies identified neurochemical changes in the CNS of GF animals. For example, compared with SPF mice, GF mice have an altered hippocampal expression of brain-derived neurotrophic factor (BDNF), but results regarding BDNF levels in GF animals are contradictory [21, 26, 31]. BDNF is an important neurotrophic factor impacting growth and survival of neurons and affecting synaptic transmission [32]. Additionally, male GF mice have elevated levels of hippocampal serotonin and increased levels of plasma tryptophan (serotonin precursor) [33]. Remarkably, microbiota was shown to regulate serotonin synthesis in the periphery [34], and GF animals have lower serotonin levels in plasma [35].

Communication pathways between the CNS and microbiota involve immunological, endocrine, metabolic, and neural pathways [36-38]. A direct neural connection between the gut and the brain is provided by the vagus nerve. Intraduodenal injection of Lactobacillus johnsonii caused increased gastral vagal nerve signaling and reduced renal sympathetic nerve activity in rats [39]. Long-term treatment with the probiotic bacterium Lactobacillus rhamnosus in conventionally colonized mice induced alterations in $\gamma$-aminobutryic acid mRNA expression in specific brain regions and reduced anxiety and depression-related behavior, which was mediated by 
Table 1 Differences in central nervous system functions between germ-free (GF) and conventionally colonized mice

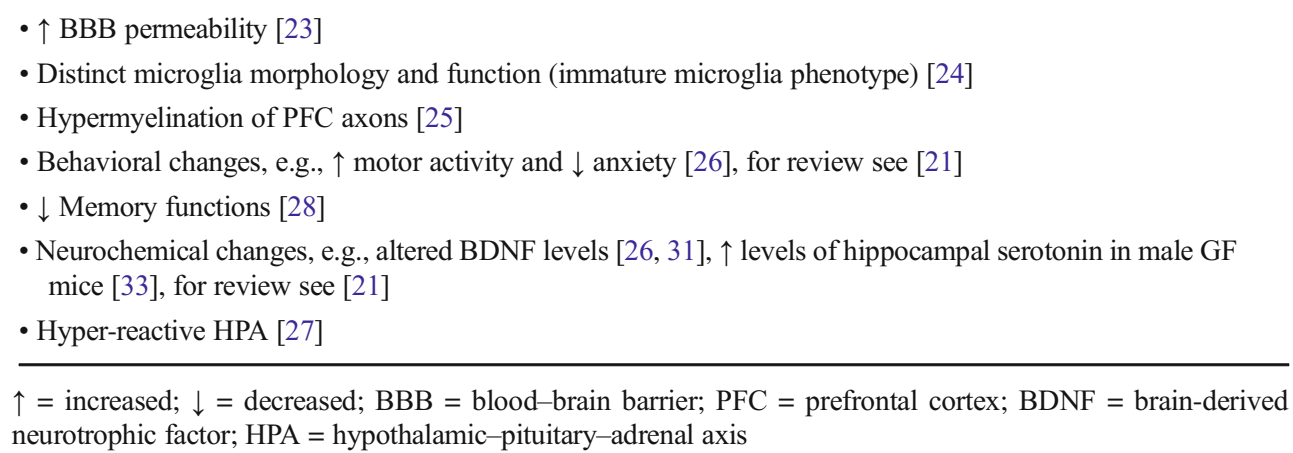

- Distinct microglia morphology and function (immature microglia phenotype) [24]

- Hypermyelination of PFC axons [25]

- Behavioral changes, e.g., $\uparrow$ motor activity and $\downarrow$ anxiety [26], for review see [21]

$\downarrow$ Memory functions [28] mice [33], for review see [21]

$\uparrow=$ increased; $\downarrow=$ decreased; BBB = blood-brain barrier; PFC
neurotrophic factor; HPA = hypothalamic-pituitary-adrenal axis

the vagus nerve [40]. Anxiolytic effects have been ascribed to Bifidobacterium longum and were apparently mediated by the vagus nerve in a mouse model of chemically induced colitis [41]. The importance of other communication pathways has been suggested, as, in a mouse model, antibiotic-induced changes in behavior were independent of vagotomy or sympathectomy [29]. Disturbances in either of the brain-gut microbiota axis pathways might contribute to the development or modulate the course of CNS disorders.

\section{CNS Disorders and Microbiota: Experimental Studies}

Links between microbiota and CNS diseases have been mainly investigated in rodent models of CNS disorders (Table 2) [21, 37, 42-44], taking the advantage of GF animals. One of the first reports on the role of gut microorganisms for the development of CNS disease originated from the field of studies in an experimental autoimmune encephalomyelitis (EAE) mouse model of multiple sclerosis (MS). Inflammatory T cellinduced destruction of the myelin sheath is a key mechanism in MS pathobiology. Notably, GF mice develop an attenuated form of EAE or even no disease, compared with conventionally colonized mice $[45,46]$. This has been attributed to changes in the balance between proinflammatory $\mathrm{T}$ helper 17 and anti-inflammatory $\mathrm{T}$ regulatory (Tregs) lymphocytes, which is shifted towards protective Tregs in GF animals. Additionally, it has been shown that dendritic cells from GF mice have a reduced ability to elicit proinflammatory responses [46]. In conventionally colonized hosts, gut microbiota has also been identified as important disease modifier in the EAE model. 2D2 myelin oligodendrocyte glycoproteinspecific T cell transgenic mice are prone to develop EAE after immunization [47]. Interestingly, susceptibility for EAE in tumor necrosis factor receptor 2 knockout mice crossed with 2D2 mice is gender specific and associated with distinct microbiota patterns. Females are more prone to EAE, whereas males, having different microbiome composition, are EAE resistant. Additionally, antibiotic treatment ameliorates disease, suggesting a crucial role of microbiota in the development of EAE [48]. Furthermore, EAE has been linked to the dysfunction in the intestinal barrier [49]. In a very recent report, a connection between dietary tryptophan, metabolized by microbiota to aryl hydrocarbon receptor ligands and severity of EAE was established. Tryptophan reduced CNS inflammation over modulation of astrocyte activity, which was mediated by aryl hydrocarbon receptor [50].

Interestingly, the therapeutic potential of the microbiota does not appear to depend on the whole microbiota or on specific subpopulations, but rather on several or even single bacterial molecule. For example, the capsular polysaccharide from Bacteroides fragilis protects mice from the development of induced EAE, correlating with a shifted balance in the CD4+ cells populations towards interleukin (IL)-10-producing CD4+ FoxP3 Treg cells [51]. Moreover, polysaccharide from B. fragilis influences the maturation of the immune answer and regulates the balance between $\mathrm{Th} 1$ and $\mathrm{Th} 2$ responses [52].

Beneficial effects of $B$. fragilis have also been investigated in the murine maternal immune activation (MIA) model. MIA shares common features with autism spectrum disorder in humans. In this model, pregnant mice are injected with a synthetic poly (I:C) double-stranded RNA mimicking viral infection. The offspring of poly I:C-injected mice display stereotypic behavior and deficits in social interaction and communication. They have altered, dysbiotic, composition of microbiota, accompanied by increased intestinal permeability and distinct intestinal cytokine profiles. Treatment of MIA mice with $B$. fragilis helps to rebalance the composition of microbiota, improves integrity of the epithelial barrier, and reverses behavioral abnormalities [53].

In the field of studies on neuropsychiatric diseases, experimental data suggest involvement of the microbiota in the pathogenesis of eating disorders [54]. Furthermore, altered microbiota composition has been reported in the mouse model of depression [55]. In rodent models, early-life stress alters gut microbiota composition [56, 57], and both stress-induced pathophysiological changes in the host and gut microbiota are necessary for the induction of anxiety-like behavior [57]. Evidence from experimental models suggests involvement of gut microbiota in visceral pain syndromes [37].

Microbiota might also contribute to the development of neurodegenerative CNS disorders, which has been demonstrated in a mouse model of Alzheimer's disease [58, 59]. 
Table 2 Summary of experimental and clinical studies on gut microbiota in neurological and neuropsychiatric diseases

Studies Mouse

$\begin{array}{ll}\text { EAE } & \text { Only mild form of EAE or no disease after EAE induction } \\ \text { MS } & \text { in GF mice, linked with shifts in the Th17/Treg balance } \\ & \text { and } \downarrow \text { DC functions }[45,46] \\ & \uparrow \text { Relative abundance of species Bacteroides acidifaciens, } \\ & \text { Bacteroides ovatus, Akkermansia muciniphila, } \uparrow \text { relative } \\ & \text { abundance of } \text { Oscillospira, Anaeroplasma } \text { and Sutterella } \\ & \text { in male TNFR2-ko 2D2 transgenic mice resistant to EAE } \\ & \text { compared with disease-susceptible females [48] } \\ & \text { PSA from B. fragilis ameliorates EAE symptoms when } \\ & \text { given as therapy or prevention [51] }\end{array}$

Eating disorders

- Possible role of autoantibodies triggered by bacterial proteins in the pathogenesis [54]

Depression

$\mathrm{AD}$

Stroke

- Different microbiota composition in mice after bilateral olfactory bulbectomy (experimental model of depression) as compared with sham-operated mice [55]

- Microbiota essential for the characteristic behavioral phenotype after maternal separation in the mouse model of depression [57]

- $\downarrow$ Amyloid $\beta$ pathology in GF AD mice [58]

- Antibiotic-induced dysbiosis linked with $\downarrow$ amyloid pathology and $\downarrow$ gliosis in AD mouse model [59]

- Different microbiota composition in stroke mice compared with sham-operated and naïve animals, $\uparrow$ relative abundance of Peptococcaeae, and $\downarrow$ relative abundance of Prevotellaceae correlating with lesion severity [15]

- $\downarrow \alpha$-diversity with several genera altered, $\downarrow$ intestinal motility after stroke; proinflammatory immune cells infiltrating the brain originate from the intestine; fecal transplant with balanced microbiota has neuroprotective effects [16]

- $\downarrow$ Lesion volume via downregulation of IL-17 $\gamma \delta$ T cells in mice with dysbiotic microbiota [64]

- $\uparrow$ Mortality after stroke after extensive depletion of microbiota by antibiotic pretreatment [67]

- Worse MCAo long-term outcome after microbiota transplantation from an aged host [60]

- $\downarrow$ Neuronal injury and $\uparrow$ cognitive performance after Clostridium butyricum treatment in bilateral common carotid occlusion in diabetic mice (ischemia/reperfusioninduced brain injury) [70]

TBI
- Correlation of TBI severity with changes in Bacteroidetes, Bacteroidetes family, Porphyromonadaceae, Firmicutes, and Proteobacteria [15]
- $\uparrow$ Abundance of genera (Pseudomonas, Mycoplana, Haemophilus, Blautia, Dorea) in patients with MS compared with healthy controls $\uparrow$ Abundance of Parabacteroides, Adlercreutzia, and Prevotella in controls [73]

- $\uparrow$ Relative abundance of microorganisms from genera pf Methanobrevibacter and Akkermansia, $\downarrow$ relative abundance of Butyricimonas correlating with changes in genes regulating immune response, $\downarrow$ abundance of Collinsella, Slackia, and Prevotella, $\uparrow$ breath methane concentrations in untreated patients with MS compared with healthy controls; treatment linked with alterations in the microbiome composition [74]

- Microbiome of anorexia nervosa patients $v s$ healthy controls: $\downarrow \alpha$-diversity, $\uparrow$ Bacilli class, unspecified genus in Coriobacteriales family $\downarrow$ Clostridia class, order Clostridiales, genera Anaerostipes and Faecalibacterium; changes in microbiota with weight restoration [136]

- $\uparrow \alpha$-diversity and alterations in several bacterial groups of gut microbiota in patients with active major depressive disorder compared with healthy controls, e.g., $\uparrow$ relative abundance of genera Alistipes, Blautia, Clostridium XIX, Lachnospiracea incertae sedis, Megamonas, Parabacteroides, Parasutterella, Phascolarctobacterium, Oscillibacter, and Roseburia in patients; $\uparrow$ abundance of genera Bacteroides, Dialister, Faecalibacterium, Prevotella, and Ruminococcus in healthy controls [111]

- Dysbiotic microbiome in patients with stroke and TIA: $\uparrow$ genera Enterobacter, Megasphaera, Oscillibacter, and Desulfovibrio; $\downarrow$ genera Bacteroides, Prevotella, and Faecalibacterium correlating with disease severity. $\downarrow$ TMAO in stroke/TIA group compared with patients with asymptomatic atherosclerosis [14]

- $\downarrow$ Proportion of Roseburia, Bacteroides, and Faecalibacterium prausnitzii in acute stroke compared with healthy controls and patients with irritable bowel syndrome, $\uparrow$ proportion of Enterobacteriaceae, Bifidobacteriaceae, and Clostridium difficile compared with healthy controls; specific temporal changes of microbial make-up after stroke with restoration after a few weeks [75]

- Different microbiota composition in patients with symptomatic atherosclerosis (minor brain infarction $=$ no severe functional deficits, TIA, occlusion of the retinal artery) compared with healthy controls: $\uparrow$ Collinsella in atherosclerosis patients, whereas $\uparrow$ Roseburia, Eubacterium, and 3 species of Bacteroides in controls [81]

- No differences in the composition of gut microbiome between symptomatic atherosclerosis patients (minor ischemic stroke, TIA, amaurosis fugax) and controls; several bacterial groups originating possibly from the gut microbiota detected in the atherosclerotic plaques; correlation between microbiome composition and lipid blood profile [78]

- Differences in gut microbiota composition in patients with SCI compared with healthy controls: $\downarrow$ total 
Table 2 (continued)

\begin{tabular}{ll}
\hline Studies & counts of bacteria from genera Pseudobutyrivibrio, \\
& $\begin{array}{l}\text { Dialister, and Megamonas in patients with UMN } \\
\text { bowel syndrome; } \downarrow \text { total counts of microorganisms } \\
\text { from genera Roseburia, Pseudobutyrivibrio, and }\end{array}$ \\
& $\begin{array}{l}\text { Megamonas in patients with LMN bowel syndrome; } \\
\text { UMN vs LMN } \downarrow \text { Marvinbryantia } \text { [12] }\end{array}$ \\
PD & $\begin{array}{l}\downarrow \text { Abundance of Prevotellaceae in patients with } \\
\text { PD compared with controls; specific bacterial } \\
\text { groups correlating with motor phenotype [11] }\end{array}$ \\
GBS & $\begin{array}{c}\text { Involvement of Campylobacter jejuni in the } \\
\text { pathogenesis postulated [43] }\end{array}$ \\
ASD & $\begin{array}{l}\text { Several studies reporting microbiota changes in } \\
\text { children with ASD without consistent results, } \\
\text { reviewed in [137] }\end{array}$
\end{tabular}

$\mathrm{EAE}=$ experimental autoimmune encephalomyelitis; $\mathrm{MS}=$ multiple sclerosis; $\mathrm{GF}=$ germ-free; $\mathrm{Th}=\mathrm{T}$ helper; Treg $=\mathrm{T}$ regulatory DC = dendritic cell; TNFR2-ko = tumor necrosis factor receptor 2 knockout; PSA = polysaccharide; $\mathrm{AD}=$ Alzheimer's disease; $\mathrm{IL}=$ interleukin; $\mathrm{MCAo}=$ middle cerebral artery occlusion; TIA = transient ischemic attack; TMAO = trimethylamine $\mathrm{N}$-oxide; $\mathrm{TBI}=$ traumatic brain injury; $\mathrm{SCI}=$ spinal cord injury; $\mathrm{UMN}=$ upper motor neuron; LMN = lower motor neuron; PD = Parkinson's disease; GBS = Guillain-Barré syndrome; ASD = autism spectrum disorder

\section{Stroke and Microbiota: Experimental Studies}

To date, only a few experimental studies focusing on the role of gut microbiota in cerebral ischemia have been published. Microbiota transferred from an aged host might contribute to deterioration of functional long-term outcome in a mouse model of focal cerebral ischemia [middle cerebral artery occlusion (MCAo)] [60]. However, the exact mechanisms involved in this process are not known [60]. In a rat MCAo model, bacteria translocated from the gut to extraintestinal organs after stroke, which might trigger systemic inflammatory response or even cause poststroke infections [61]. Additionally, stress before stroke might boost bacterial translocation from the intestine into the bloodstream [62]. A recent study demonstrated alterations in microbiota profile after severe cerebral ischemia and linked poststroke dysbiosis with induction of proinflammatory immune response. Transplantation of balanced microbiota after cerebral ischemia improved stroke outcome [16].

In another report antibiotic-induced gut dysbiosis with significantly decreased $\alpha$-diversity of gut microbiota (diversity within particular habitat [63]) improved outcome and limited the size of ischemic cerebral lesion as measured histologically 3 days after MCAo. These effects were attributed to a decrease in IL-17 producing $\gamma \delta \mathrm{T}$ cells and an increase in Treg cells in the small intestine, and consequently limited infiltration of harmful IL-17 $+\gamma \delta$ T cells to the meninges [64].

Interestingly, similar mechanisms were suggested to explain the effects of high-fat diet on the pathogenesis of type 2 diabetes and obesity. A high-fat diet induced dysbiotic changes in the gut microbiota, impaired function of antigen presenting cells, and decreased number of IL-17 producers, ROR $\gamma \mathrm{t} \mathrm{CD} 4+\mathrm{T}$ cells, in the intestine. These alterations preceded the onset of metabolic disease [65]. Obesity and diabetes are, however, well-established risk factors for stroke [66].

Our group investigated the outcome of experimental stroke in C57BL/6 mice after extensive microbiota depletion with broad-spectrum antibiotic pretreatment [67]. We observed significantly increased mortality in microbiota-depleted animals when the antibiotics were stopped before induction of cerebral ischemia. Surprisingly, mortality was linked to acute severe colitis. This phenotype was rescued upon colonization with SPF microbiota or continuous antibiotic treatment. These observations underline the importance of the complex microbial community after cerebral ischemia, when the host immune system is severely compromised by stroke-induced immunodepression [68], which affects immune barriers even in the intestine [69]. We did not observe any effects of microbiota depletion on infarct volume in the brain 1 day after MCAo [67].

Furthermore, model-specific changes in the microbiota after murine MCAo and mild traumatic brain injury were described very recently. In MCAo animals alterations in Peptococcaceae and Prevotellaceace correlated with infarct severity. Additionally, inducing focal cerebral ischemia in mice increased noradrenaline release in the gastrointestinal tract (cecum), and reduced the number of mucoproteinproducing cells and goblet cells. Increased noradrenaline levels and changes in goblet cell function may directly affect the gut microbial community after stroke [15].

A study in diabetic mice identified beneficial effects of supplementation with Clostridium butyricum in ischemia/ 
reperfusion-induced brain injury after bilateral common carotid artery occlusion. Treatment with $C$. butyricum decreased neuronal injury and improved the cognitive functions [70]

\section{Clinical Data}

Clinical data on the brain-gut microbiota connection are still scarce (Table 2). Hitherto, clinical microbiota research focused on gastrointestinal, nutritional, or endocrine disorders such as inflammatory bowel disease, obesity, or diabetes type 2 .

First clinical reports on the role of gut microbiota indisorders of the nervous system investigated alterations in the composition of the gut microbiome with neurological disease but did not provide a causative link. It was consequently conjectured that gut microbiota plays a role in such different conditions as PD, visceral pain, Guillain-Barré syndrome, stroke, and psychiatric diseases [42, 43, 71]. Changes in the composition of gut microbiota have already been reported in PD. Patients with PD had decreased abundance of Prevotellaceae in their microbiota compared with control subjects. Increased abundance of Enterobacteriaceae was correlated with a more severe motor phenotype [11]. It is further hypothesized that gut microbiota may also play a role in nonmotor symptoms of PD such as neuropsychiatric conditions, sleep disturbances, and pain syndromes [72].

Spinal cord injury is another CNS disorder where the composition of microbiota has recently been investigated. Patients with spinal cord injury have fewer butyrate-producing bacteria in the gut; the consequences of this has not yet been clarified [12]. Moreover, alterations in gut microbiota profiles were observed in patients with MS [73, 74]. Interestingly, immunomodulatory treatment in MS had also effects on the microbiota composition [74].

Changes in the composition of microbiota in the course of stroke were already reported [75], but more importantly, microbiome might contribute to the pathogenesis of the disease, influencing formation of atherosclerotic plaque, as suggested by experimental, as well as clinical data [76-78]. Microbiota is involved in the metabolism of phosphatidylcholine. Levels of its metabolites choline, betaine, and, in particular, trimethylamine $\mathrm{N}$-oxide (TMAO; host product of trimethylamine oxidation), have been identified as predictors of cardiovascular disease risk [76, 77, 79, 80]. TMAO is also produced during the metabolism of L-carnitine, which is found in red meat [80]. All of these metabolites increase cholesterol accumulation in macrophages and promote foam cell formation [77]. Conversely, symptomatic atherosclerosis was linked to changes in the composition of gut microbiome [81].

A recent clinical study compared microbiome composition in patients diagnosed with asymptomatic atherosclerosis, stroke, and transient ischemic attack. The trial data also included patient TMAO plasma levels. Surprisingly, the asymptomatic atherosclerosis group with and without carotid plaques had similar levels of TMAO and comparable gut microbiota composition. In contrast, patients with stroke and transient ischemic attack differed substantially from the asymptomatic subjects regarding microbiome composition, but their TMAO levels were lower than those of the asymptomatic group [14].

In another study, serum levels of carnitine and its metabolite $\gamma$-butyrobetaine - rather than TMAO and trimethyllysine - were elevated in patients with carotid atherosclerosis. Serum levels of gamma-butyrobetaine and trimethyllysine were also associated with cardiovascular death [82]. Conflicting evidence on gut microbiota metabolites and atherosclerosis was also submitted by experimental studies. Atherosclerotic plaque formation was increased in apolipoprotein $\mathrm{E}$ knockout transgenic mice $\left(\mathrm{ApoE}^{-1}\right.$; mouse model of atherosclerosis [83]) when the mice were supplemented with L-carnitine [80]. ApoE ${ }^{-1-}$ animals, however, lack an important enzyme cholesteryl ester transfer protein, which transfers cholesterol ester between lipoproteins of high and low density. Interestingly $\mathrm{ApoE}^{-/}$transgenic mice overexpressing human cholesteryl ester transfer protein supplemented with Lcarnitine had decreased aortic atherosclerotic plaque formation and high TMAO levels [84]. Moreover, in contrast to conventionally colonized $\mathrm{ApoE}^{-/-}$mice, the same transgenic animals housed under GF conditions developed severe atherosclerosis when fed a low cholesterol diet [85].

These data suggest that metabolites of the microbiome might also provide a certain level of protection from the development of disease. It should be noted that gut microbiota studies in the clinical settings are more challenging than experimental studies in animals. Study populations in humans are usually more heterogeneous, as they are influenced by multiple factors independent of the disorder under investigation. These confounders might affect the composition of the intestinal microbial community as well [71]. Furthermore, as illustrated by the example of studies in atherosclerosis, microbiota may confer dualistic effects on the host - protective and harmful - in the pathogenesis and course of the disease [86] .

\section{Open Issues and Future Avenues of Research}

\section{Gut Microbiota as Therapeutic Target in Stroke}

Gut microbiota could be a potential therapeutic target for diseases that constitute risk factors for stroke and/or for complications after cerebral ischemia (Fig. 1). Experimental and clinical evidence suggests that microbiota in combination with a high-fat diet contributes to the development of type 2 diabetes [87], obesity [65, 87-91], and hypertension [92, 93]. Elevated TMAO plasma levels, linked with adverse cardiovascular events in humans, were normalized by antibiotic treatment [79]. However, prolonged antibiosis for atherosclerosis prevention is potentially harmful as it poses an increased risk of 
regulation of Th1/Th17/Treg

$\gamma \delta \mathrm{T}$ cell - responses

lesion size, outcome

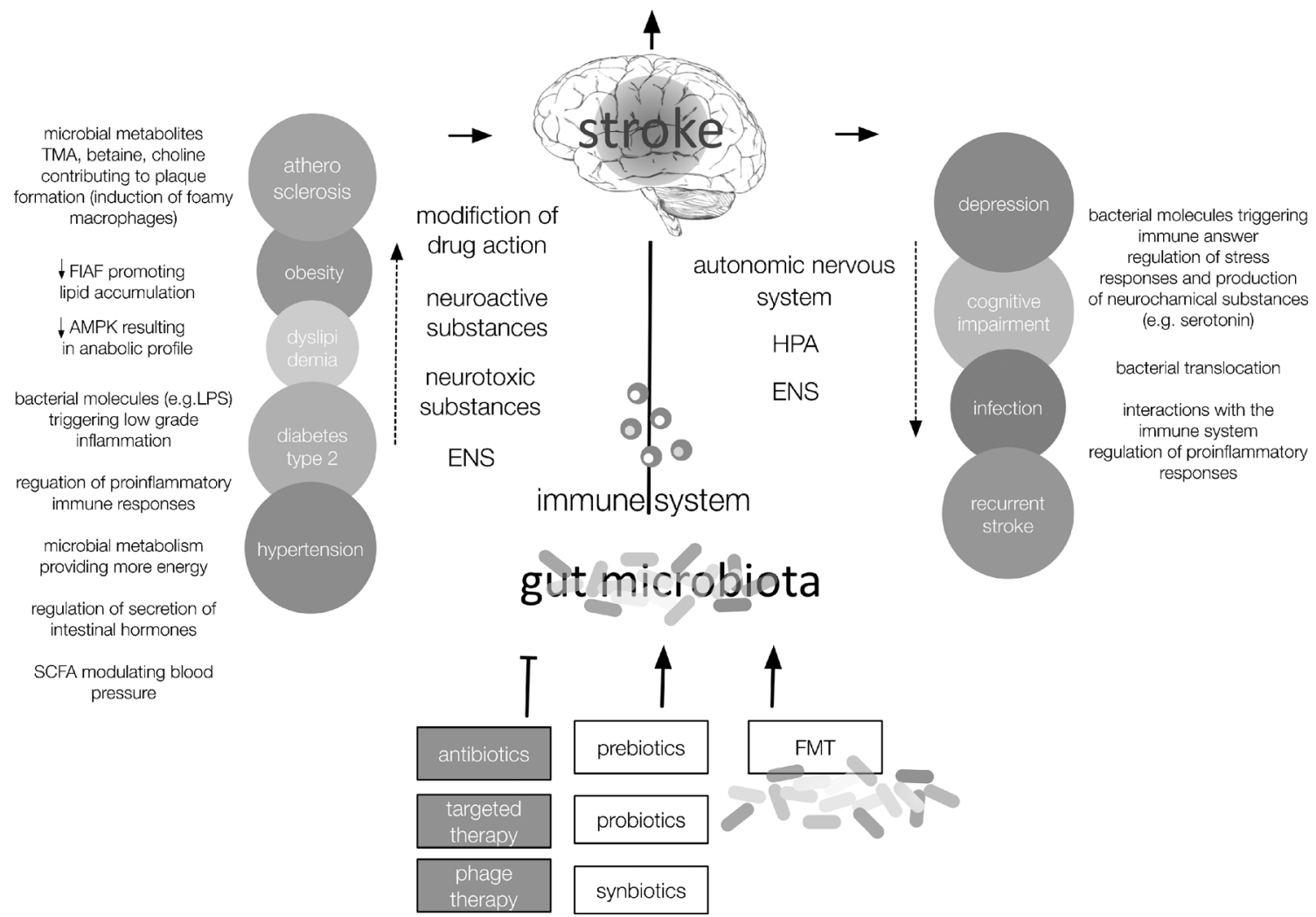

Fig. 1 Modifiable stroke risk factors and poststroke complications with hypothesized involvement of the gut microbiota. Gut microbiota might contribute to the pathogenesis of cerebrovascular disorders and serve as a therapeutic target for modulating stroke-related risk factors, neuroinflammatary responses, and complications after stroke. The interplay between the gut microbiome and the host involves the host immune system, hypothalamus-pituitary-adrenal glands axis (HPA), autonomic

antibiotic resistance and detrimental infections such as Clostridium difficile colitis. In a more sophisticated approach, TMAO production has been successfully inhibited by 3,3-dimethyl-1-butanol, a structural analog of choline and an antagonist for the microbial trimethylamine lyase [94].

Further, microbiome-based therapy appears to be a promising approach for the treatment of poststroke complications. Complications after cerebral ischemia comprise, among others, bacterial infections, cognitive impairment, and depression $[95,96]$. In particular, stroke-associated pneumonia is considered to contribute to detrimental neurological outcome. We would speculate that these conditions can be targeted via the gut microbiome. Although experimental findings are equivocal, gut microbiota might be the source of systemic infections in patients with stroke, particularly when host immunity and immunological barriers are compromised by poststroke immunodepression [97]. In a mouse model gut microbiota has been demonstrated to support the host immune defense against pneumococcal pneumonia nervous system, enteric nervous system (ENS), and microbial molecules and metabolites. $\mathrm{Th}=\mathrm{T}$ helper; Treg $=\mathrm{T}$ regulatory; FMT $=$ fecal microbial transplantation; FIAF $=$ fasting-induced adipose factor; $\mathrm{AMPK}=5^{\prime}$ adenosine monophosphate-activated protein kinase; LPS = lipopolysacharide; $\mathrm{TMA}=$ trimethylamine; $\mathrm{SCFA}=$ short-chain fatty acids

[98]. After microbiota depletion with antibiotic treatment, C57BL/6 mice infected with Streptococcus pneumoniae showed higher mortality, more severe organ damage, increased spread of the pathogen, elevated inflammatory markers, and limited alveolar macrophages functions than did untreated mice. Transfer of fecal microbiota from untreated animals reduced bacterial counts in the lungs and normalized inflammatory markers. [98]. Whether the gut microbiota or rather the microbiota of the respiratory tract is responsible for these effects remains a matter of debate. Both microbial communities are affected by the antibiotic treatment used in this study to deplete the microbiota, as well as by fecal transplantation over oral gavage, implemented to restore microbial community [99].

In current practice patients with stroke are often treated with antibiotics in the acute course of the disease due to poststroke infections. Certainly, antibiotic treatment will target not only pathogens in lung and urinary tract, but also the commensal bacterial populations in other organs such as the 
gut. Antibiotics induce rapid and long-lasting changes in the microbiome [100]. Treatment with clindamycin over 7 days triggered shifts in the microbiota, detected even 2 years after the termination of the antibiotic therapy [101]. Patients with severe strokes are usually treated for 5 to 10 days and often with combinations of broad-spectrum antimicrobial agents [102]. In experimental stroke, profound depletion of microbiota by extensive broad-spectrum antibiotic pretreatment to eliminate cultivatable bacterial microorganisms causes detrimental outcome due to acute and severe colitis [67].

Investigations in an animal model [103], as well as in a clinical study [104], suggest that stroke triggers a metabolic switch to a catabolic profile, causing sarcopenia and substantial weight loss. Although the underlying mechanisms are not fully elucidated, involvement of commensal bacteria is likely. It has been suggested that shifts in microbiota are associated with weight changes [105]. Altered composition of gut microbiota has been observed in a mouse model of acute leukemia accompanied by cachexia [106]. Restoration of specific microbial species led to reduction of the inflammatory cytokines and markers involved in the protein breakdown in skeletal muscle [106].

Stroke survivors often suffer from neuropsychiatric complications, mainly poststroke depression [95]. It has been shown that a multitude of mechanisms, including systemic inflammatory processes, are involved in the manifestation of poststroke depression [107]. However, it is also reasonable to assume a role for gut microbiota, as experimental and clinical studies suggest a causative role of microbiota in mood disorders [108-111].

The abovementioned relations are largely speculative but ultimately testable. They provide examples for how gut microbiome might serve as target to treat or even prevent conditions that pose risk factors for stroke or poststroke complications.

\section{Therapeutic Manipulation of the Microbiome?}

Principally, there are two strategies for manipulating microbiota: 1) target a defined microorganism by direct elimination, modification, or triggering of the immune response via vaccination with a specific epitope; and 2) manipulate whole communities either individually or by combinations of antibiotics, probiotics, prebiotics, synbiotics, or fecal transplant [112].

Targeting specific microbiota is promising when a causal link has been demonstrated between a particular microorganism and a disease. The approach of targeting whole communities appears to be a shot in the dark: the outcome of such a therapy is not only unpredictable, but might also have more severe adverse effects, in particular when the entire microbial community is profoundly influenced. As already discussed, antibiotic treatment might shift the gut microbiome incurring detrimental outcome, in particular in immune compromised patients with stroke. In contrast, several probiotics have been shown to exert beneficial effects on the human immune system [113], while difficulties can and do arise owing to the fact that some bacterial strains are not even able to survive the human upper gastrointestinal tract, and often do not colonize the gut. The choice of which probiotic strain to use as a treatment is therefore complicated. Commonly, probiotic supplementation is considered to be well tolerated. However, treatment with probiotics might have harmful consequences. For example, the PROPATRIA (PRObiotics in PAncreatitits TRIAl) trial had to be stopped prematurely owing to significantly higher mortality in the group treated with probiotics $[114,115]$. Moreover, several immunocompromised patients or newborns are reported to have suffered from sepsis due to probiotic microorganisms [116-118]. These findings advise caution, given that stroke induces an immune depression [68].

Ever since 1958, transfer of a whole microbiota community from a healthy donor, so-called fecal microbiota transplantation (FMT), has been established in the treatment of patients with severe $C$. difficile colitis. At that time the causative agent had not even been identified [119]. The effectiveness of FMT in pseudomembranous colitis was recently confirmed by a clinical study comparing FMT with vancomycin treatment [120]. However, the optimal way of delivering the "transplant" to the gut has not yet been established. Studies are being carried out to improve fecal bacteriotherapy in order to recolonize the gut with microorganisms from healthy donors [121]. Delivering fecal microbiota by pill allows standardization and might reduce risks associated with the more invasive delivery [122]. However, standards for identifications of persons who qualify as healthy donors are also not yet fully established. Since the field of microbial therapy is in its infancy and little is known about side effects, transplantation of currently unknown pathogenic microorganisms by FMT cannot be excluded. Some undesirable and hitherto unpredictable effects of FMT have been already reported, for example peripheral neuropathy [123]. Moreover, in the experimental setting, transplantation of intestinal microbiota from an atherosclerosis-prone to a disease-resistant mouse strain was sufficient to transmit susceptibility to atherosclerosis [124]. Thus, even before risk factors for diseases manifest, gut microbiota might have pathogenic potential, making the definition of healthy persons as donors for FMT inherently difficult. Nonetheless, stool transplant has already been approved in USA by the Federal Drug Administration for treating $C$. difficile enteritis that is nonresponsive to standard therapies; however, the legal status of FMT is still being debated [125]. Furthermore, novel therapeutic approaches, specifically targeting microbiome are developing rapidly, including phage therapy, targeting quorum-sensing molecules, or certain bacterial genes $[100,126]$.

High expectations for developing new microbiota-based therapies are pinned on the microbial interplay with the immune system and the metabolic capacity of the microbiome. 
Gut microbiota possesses metabolic capacities comparable with those of a human liver [127]. Products of bacterial fermentation, short-chain fatty acids, modulate immune responses [128], and might contribute to the pathogenesis of hypertension [93]. Moreover, gut bacteria can directly metabolize drugs to influence their activity or indirectly modify host responses to xenobiotics [129]. This has implications for the bioavailability of drugs and their toxicity. For example, levodopa, used in the treatment of PD, needs to be decarboxylated to dopamine in the CNS. Intestinal bacteria are also capable of decarboxylating the drug [130]. However, levodopa, but not dopamine, is able to cross the blood-brain barrier, so decarboxylation outside the CNS would affect its brain availability [131].

In an experimental mouse study, nonlethal inhibition of bacterial $\beta$-glucuronidases, which reactivate the colon cancer drug CPT-11 (Irinotecan), lead to alleviation of the drug's toxicity [132]. These effects seem to depend on a specific composition of gut bacteria. Gut microbiota (and, specifically, Bacteroides species) has proved to be indispensable for efficacy of certain anticancer drugs (Ipilimumab, anti-cytotoxic T-lymphocyte-associated protein 4), which are unsuccessful in GF or antibiotic-treated mice [133]. Bifidobacterium promoted success of antitumor therapy with anti-programmed celldeath-ligand 1 by boosting T-cell responses [134].

Taken together, composition of gut microbiota might influence treatment success of certain drugs through its microbial metabolic functions or by modulating the immune response. This field of research certainly needs to be followed up to disentangle the therapeutic potential of the commensal microorganisms and help to predict individual responses to certain therapies. It appears very likely that many commonly used drugs, not investigated in the context of gut microbial composition, might exert their effects, at least in part, on microorganisms of the gut.

One of the main obstacles in current microbiome-based treatment research is the definition of measurable and clinically relevant microbiota-related endpoints for predicting the success of the therapy [135]. There is no generally accepted method or even gold standard for monitoring microbiota that could be implemented in the clinical setting. More importantly, relevant endpoints for efficacy, comparable with clinical trials investigating drugs for treatment, need to be used to prove a causative link between a certain microbiota composition and a particular condition, and to provide a novel microbiome-based treatment strategy [135].

In summary, gut microbiota research is a flourishing field with novel and often surprising discoveries about the role of commensal microorganisms in health and disease. Thanks to the advances in sampling and sequencing techniques, the detailed characterization and profiling of microbiome is now feasible. Although the concept of gut microbiota-brain communication seems to be established now, investigations are still needed to characterize the mechanistic connections between changes in the gut microbiota and neurological diseases. This would be the prerequisite for developing successful microbiota-based therapies in CNS disorders, the effectiveness and safety of which would need to be tested in experimental studies and large clinical trials. Although most of this research has been done outside neurosciences, we provided examples, which we believe to be instructive for stroke research.

Required Author Forms Disclosure forms provided by the authors are available with the online version of this article.

\section{Compliance with Ethical standards}

Funding This work was supported by the German Research Foundation (Exc 257) and the Federal Ministry of Education and Research (01 EO 08 01). KW received $\mathrm{PhD}$ scholarship from the International Max Planck Research School for Infectious Diseases and Immunology (IMPRS-IDI) and the Sonnenfeld-Stiftung. We thank Ms. Catherine Aubel for proofreading the manuscript.

Open Access This article is distributed under the terms of the Creative Commons Attribution 4.0 International License (http:// creativecommons.org/licenses/by/4.0/), which permits unrestricted use, distribution, and reproduction in any medium, provided you give appropriate credit to the original author(s) and the source, provide a link to the Creative Commons license, and indicate if changes were made.

\section{References}

1. Rettger, L.F., A Treatise on the Transformation of the Intestinal Flora. London: Forgotten Books. (Original work published 1921). (2013).

2. Shulman, S.T., Friedmann, H.C., and Sims, R.H., Theodor Escherich: the first pediatric infectious diseases physician? Clin Infect Dis, 2007. 45(8): p. 1025-9.

3. Grice, E.A. and Segre, J.A., The human microbiome: our second genome. Annu Rev Genomics Hum Genet, 2012. 13: p. 151-70.

4. $\mathrm{Xu}, \mathrm{J}$. and Gordon, J.I., Honor thy symbionts. Proc Natl Acad Sci U S A, 2003. 100(18): p. 10452-9.

5. O'Hara, A.M. and Shanahan, F., The gut flora as a forgotten organ. EMBO Rep, 2006. 7(7): p. 688-93.

6. Borre, Y.E., O'Keeffe, G.W., Clarke, G., Stanton, C., Dinan, T.G., and Cryan, J.F., Microbiota and neurodevelopmental windows: implications for brain disorders. Trends Mol Med, 2014. 20(9): p. 509-18.

7. Butto, L.F. and Haller, D., Dysbiosis in intestinal inflammation: Cause or consequence. Int J Med Microbiol, 2016.

8. Karlsson, F.H., Tremaroli, V., Nookaew, I., et al., Gut metagenome in European women with normal, impaired and diabetic glucose control. Nature, 2013. 498(7452): p. 99-103.

9. Bultman, S.J., Emerging roles of the microbiome in cancer. Carcinogenesis, 2014. 35(2): p. 249-55.

10. Mayer, E.A., Knight, R., Mazmanian, S.K., Cryan, J.F., and Tillisch, K., Gut microbes and the brain: paradigm shift in neuroscience. J Neurosci, 2014. 34(46): p. 15490-6. 
11. Scheperjans, F., Aho, V., Pereira, P.A., et al., Gut microbiota are related to Parkinson's disease and clinical phenotype. Mov Disord, 2015. 30(3): p. 350-8.

12. Gungor, B., Adiguzel, E., Gursel, I., Yilmaz, B., and Gursel, M., Intestinal Microbiota in Patients with Spinal Cord Injury. PLoS One, 2016. 11(1): p. e0145878.

13. De Angelis, M., Francavilla, R., Piccolo, M., De Giacomo, A., and Gobbetti, M., Autism spectrum disorders and intestinal microbiota. Gut Microbes, 2015. 6(3): p. 207-13.

14. Yin, J., Liao, S.X., He, Y., et al., Dysbiosis of Gut Microbiota With Reduced Trimethylamine-N-Oxide Level in Patients With LargeArtery Atherosclerotic Stroke or Transient Ischemic Attack. J Am Heart Assoc, 2015. 4(11).

15. Houlden, A., Goldrick, M., Brough, D., et al., Brain injury induces specific changes in the caecal microbiota of mice via altered autonomic activity and mucoprotein production. Brain Behav Immun, 2016.

16. Singh, V., Roth, S., Llovera, G., et al., Microbiota Dysbiosis Controls the Neuroinflammatory Response after Stroke. J Neurosci, 2016. 36(28): p. 7428-40.

17. O'Toole, P.W. and Claesson, M.J., Gut microbiota: Changes throughout the lifespan from infancy to elderly. International Dairy Journal, 2010. 20(4): p. 281-291.

18. Claesson, M.J., Cusack, S., O'Sullivan, O., et al., Composition, variability, and temporal stability of the intestinal microbiota of the elderly. Proc Natl Acad Sci U S A, 2011. 108 Suppl 1: p. 4586-91.

19. Reardon, S., Microbiome therapy gains market traction. Nature, 2014. 509(7500): p. 269-70.

20. Garber, K., Drugging the gut microbiome. Nat Biotechnol, 2015. 33(3): p. 228-31.

21. Luczynski, P., McVey Neufeld, K.A., Oriach, C.S., Clarke, G., Dinan, T.G., and Cryan, J.F., Growing up in a Bubble: Using Germ-Free Animals to Assess the Influence of the Gut Microbiota on Brain and Behavior. Int J Neuropsychopharmacol, 2016.

22. Collins, J., Borojevic, R., Verdu, E.F., Huizinga, J.D., and Ratcliffe, E.M., Intestinal microbiota influence the early postnatal development of the enteric nervous system. Neurogastroenterol Motil, 2014. 26(1): p. 98-107.

23. Braniste, V., Al-Asmakh, M., Kowal, C., et al., The gut microbiota influences blood-brain barrier permeability in mice. Sci Transl Med, 2014. 6(263): p. 263ra158.

24. Erny, D., Hrabe de Angelis, A.L., Jaitin, D., et al., Host microbiota constantly control maturation and function of microglia in the CNS. Nat Neurosci, 2015. 18(7): p. 965-77.

25. Hoban, A.E., Stilling, R.M., Ryan, F.J., et al., Regulation of prefrontal cortex myelination by the microbiota. Transl Psychiatry, 2016. 6: p. e774.

26. Heijtz, R.D., Wang, S., Anuar, F., et al., Normal gut microbiota modulates brain development and behavior. Proceedings of the National Academy of Sciences of the United States of America, 2011. 108(7): p. 3047-52.

27. Sudo, N., Chida, Y., Aiba, Y., et al., Postnatal microbial colonization programs the hypothalamic-pituitaryadrenal system for stress response in mice. J Physiol, 2004. 558(Pt 1): p. 263-75.

28. Gareau, M.G., Wine, E., Rodrigues, D.M., et al., Bacterial infection causes stress-induced memory dysfunction in mice. Gut, 2010.

29. Bercik, P., Denou, E., Collins, J., et al., The Intestinal Microbiota Affect Central Levels of Brain-Derived Neurotropic Factor and Behavior in Mice. Gastroenterology, 2011.

30. Bruce-Keller, A.J., Salbaum, J.M., Luo, M., et al., Obese-type gut microbiota induce neurobehavioral changes in the absence of obesity. Biol Psychiatry, 2015. 77(7): p. 607-15.

31. Neufeld, K.M., Kang, N., Bienenstock, J., and Foster, J.A., Reduced anxiety-like behavior and central neurochemical change in germ-free mice. Neurogastroenterol Motil, 2011.23(3): p. 25564, e119.

32. Binder, D.K. and Scharfman, H.E., Brain-derived neurotrophic factor. Growth Factors, 2004. 22(3): p. 123-31.

33. Clarke, G., Grenham, S., Scully, P., et al., The microbiome-gutbrain axis during early life regulates the hippocampal serotonergic system in a sex-dependent manner. Mol Psychiatry, 2012.

34. Yano, J.M., Yu, K., Donaldson, G.P., et al., Indigenous bacteria from the gut microbiota regulate host serotonin biosynthesis. Cell, 2015. 161(2): p. 264-76.

35. Wikoff, W.R., Anfora, A.T., Liu, J., et al., Metabolomics analysis reveals large effects of gut microflora on mammalian blood metabolites. Proc Natl Acad Sci U S A, 2009. 106(10): p. 3698-703.

36. Collins, S.M., Surette, M., and Bercik, P., The interplay between the intestinal microbiota and the brain. Nat Rev Microbiol, 2012. 10(11): p. 735-42.

37. Cryan, J.F. and Dinan, T.G., Mind-altering microorganisms: the impact of the gut microbiota on brain and behaviour. Nat Rev Neurosci, 2012. 13(10): p. 701-12.

38. Rhee, S.H., Pothoulakis, C., and Mayer, E.A., Principles and clinical implications of the brain-gut-enteric microbiota axis. Nat Rev Gastroenterol Hepatol, 2009. 6(5): p. 306-14.

39. Tanida, M., Yamano, T., Maeda, K., Okumura, N., Fukushima, Y., and Nagai, K., Effects of intraduodenal injection of Lactobacillus johnsonii La1 on renal sympathetic nerve activity and blood pressure in urethaneanesthetized rats. Neurosci Lett, 2005. 389(2): p. 109-14.

40. Bravo, J.A., Forsythe, P., Chew, M.V., et al., Ingestion of Lactobacillus strain regulates emotional behavior and central GABA receptor expression in a mouse via the vagus nerve. Proc Natl Acad Sci U S A, 2011. 108(38): p. 16050-5.

41. Bercik, P., Park, A.J., Sinclair, D., et al., The anxiolytic effect of Bifidobacterium longum NCC3001 involves vagal pathways for gut-brain communication. Neurogastroenterol Motil, 2011. 23(12): p. 1132-9.

42. Ochoa-Reparaz, J., Mielcarz, D.W., Begum-Haque, S., and Kasper, L.H., Gut, bugs, and brain: Role of commensal bacteria in the control of central nervous system disease. Ann Neurol, 2011. 69(2): p. 240-7.

43. Wang, Y. and Kasper, L.H., The role of microbiome in central nervous system disorders. Brain Behav Immun, 2014. 38: p. 1-12.

44. Mayer, E.A., Tillisch, K., and Gupta, A., Gut/brain axis and the microbiota. J Clin Invest, 2015. 125(3): p. 926-38.

45. Berer, K., Mues, M., Koutrolos, M., et al., Commensal microbiota and myelin autoantigen cooperate to trigger autoimmune demyelination. Nature, 2011. 479(7374): p. 538-41.

46. Lee, Y.K., Menezes, J.S., Umesaki, Y., and Mazmanian, S.K., Proinflammatory T-cell responses to gut microbiota promote experimental autoimmune encephalomyelitis. Proc Natl Acad Sci U S A, 2011. 108 Suppl 1: p. 4615-22.

47. Bettelli, E., Pagany, M., Weiner, H.L., Linington, C., Sobel, R.A., and Kuchroo, V.K., Myelin oligodendrocyte glycoprotein-specific $\mathrm{T}$ cell receptor transgenic mice develop spontaneous autoimmune optic neuritis. J Exp Med, 2003. 197(9): p. 1073-81.

48. Miller, P.G., Bonn, M.B., Franklin, C.L., Ericsson, A.C., and McKarns, S.C., TNFR2 Deficiency Acts in Concert with Gut Microbiota To Precipitate Spontaneous Sex-Biased Central Nervous System Demyelinating Autoimmune Disease. J Immunol, 2015. 195(10): p. 4668-84.

49. Nouri, M., Bredberg, A., Westrom, B., and Lavasani, S., Intestinal barrier dysfunction develops at the onset of experimental autoimmune encephalomyelitis, and can be induced by adoptive transfer of autoreactive T cells. PLoS One, 2014. 9(9): p. e106335.

50. Rothhammer, V., Mascanfroni, I.D., Bunse, L., et al., Type I interferons and microbial metabolites of tryptophan modulate astrocyte 
activity and central nervous system inflammation via the aryl hydrocarbon receptor. Nat Med, 2016.

51. Ochoa-Reparaz, J., Mielcarz, D.W., Wang, Y., et al., A polysaccharide from the human commensal Bacteroides fragilis protects against CNS demyelinating disease. Mucosal Immunology, 2010. 3(5): p. 487-495.

52. Mazmanian, S.K., Liu, C.H., Tzianabos, A.O., and Kasper, D.L., An immunomodulatory molecule of symbiotic bacteria directs maturation of the host immune system. Cell, 2005. 122(1): p. 107-18.

53. Hsiao, E.Y., McBride, S.W., Hsien, S., et al., Microbiota modulate behavioral and physiological abnormalities associated with neurodevelopmental disorders. Cell, 2013. 155(7): p. 1451-63.

54. Tennoune, N., Chan, P., Breton, J., et al., Bacterial ClpB heatshock protein, an antigen-mimetic of the anorexigenic peptide alpha-MSH, at the origin of eating disorders. Transl Psychiatry, 2014. 4: p. e458.

55. Park, A.J., Collins, J., Blennerhassett, P.A., et al., Altered colonic function and microbiota profile in a mouse model of chronic depression. Neurogastroenterol Motil, 2013. 25(9): p. 733-e575.

56. O'Mahony, S.M., Marchesi, J.R., Scully, P., et al., Early life stress alters behavior, immunity, and microbiota in rats: implications for irritable bowel syndrome and psychiatric illnesses. Biol Psychiatry, 2009. 65(3): p. 263-7.

57. De Palma, G., Blennerhassett, P., Lu, J., et al., Microbiota and host determinants of behavioural phenotype in maternally separated mice. Nat Commun, 2015. 6: p. 7735.

58. Harach, T.M., N.; Dutilleul, N.; Cheatham, V., et al., Reduction of Alzheimer's disease beta-amyloid pathology in the absence of gut microbiota. arXiv: $1509.02273 \mathrm{v} 2$ [q-bio.MN]

59. Minter, M.R., Zhang, C., Leone, V., et al., Antibiotic-induced perturbations in gut microbial diversity influences neuro-inflammation and amyloidosis in a murine model of Alzheimer's disease. Sci Rep, 2016. 6: p. 30028.

60. Jandzinski, M., Manipulation of the microbiome and its impact on functional recovery following ischemic stroke. Honors Scholar Theses. Paper 414, 2015: p. http://digitalcommons.uconn. edu/srhonors theses/414.

61. Tascilar, N., Irkorucu, O., Tascilar, O., et al., Bacterial translocation in experimental stroke: what happens to the gut barrier? Bratisl Lek Listy, 2010. 111(4): p. 194-9.

62. Caso, J.R., Hurtado, O., Pereira, M.P., et al., Colonic bacterial translocation as a possible factor in stressworsening experimental stroke outcome. Am J Physiol Regul Integr Comp Physiol, 2009. 296(4): p. R979-85.

63. Whittaker, R.H., Evolution and Measurement of Species Diversity. Taxon, 1972. 21(2/3 ): p. 213-251.

64. Benakis, C., Brea, D., Caballero, S., et al., Commensal microbiota affects ischemic stroke outcome by regulating intestinal gammadelta T cells. Nat Med, 2016. 22(5): p. 516-23.

65. Garidou, L., Pomie, C., Klopp, P., et al., The Gut Microbiota Regulates Intestinal CD4 T Cells Expressing RORgammat and Controls Metabolic Disease. Cell Metab, 2015. 22(1): p. 100-12.

66. Sacco, R.L., Benjamin, E.J., Broderick, J.P., et al., American Heart Association Prevention Conference. IV. Prevention and Rehabilitation of Stroke. Risk factors. Stroke, 1997. 28(7): p. 1507-17.

67. Winek, K., Engel, O., Koduah, P., et al., Depletion of Cultivatable Gut Microbiota by Broad-Spectrum Antibiotic Pretreatment Worsens Outcome After Murine Stroke. Stroke, 2016. 47(5): p. 1354-63.

68. Meisel, C., Schwab, J.M., Prass, K., Meisel, A., and Dirnagl, U., Central nervous system injury-induced immune deficiency syndrome. Nat Rev Neurosci, 2005. 6(10): p. 775-86.

69. Schulte-Herbruggen, O., Quarcoo, D., Meisel, A., and Meisel, C., Differential affection of intestinal immune cell populations after cerebral ischemia in mice. Neuroimmunomodulation, 2009. 16(3): p. 213-8.

70. Sun, J., Wang, F., Ling, Z., et al., Clostridium butyricum attenuates cerebral ischemia/reperfusion injury in diabetic mice via modulation of gut microbiota. Brain Res, 2016.

71. Winek, K., Meisel, A., and Dirnagl, U., Gut microbiota impact on stroke outcome: Fad or fact? J Cereb Blood Flow Metab, 2016. 36(5): p. 891-8.

72. Felice, V.D., Quigley, E.M., Sullivan, A.M., O'Keeffe, G.W., and O'Mahony, S.M., Microbiota-gut-brain signalling in Parkinson's disease: Implications for non-motor symptoms. Parkinsonism Relat Disord, 2016.

73. Chen, J., Chia, N., Kalari, K.R., et al., Multiple sclerosis patients have a distinct gut microbiota compared to healthy controls. Sci Rep, 2016. 6: p. 28484.

74. Jangi, S., Gandhi, R., Cox, L.M., et al., Alterations of the human gut microbiome in multiple sclerosis. Nat Commun, 2016. 7: p. 12015 .

75. Swidsinski, A.L.-B., V.; Krüger, M.; Kirsch, S., Central Nervous System and the Colonic Bioreactor: Analysis of Colonic Microbiota in Patients with Stroke Unravels Unknown Mechanisms of the Host Defense after Brain Injury. Intest Res, 2012. 10(4): p. 332-342.

76. Zhu, W., Gregory, J.C., Org, E., et al., Gut Microbial Metabolite TMAO Enhances Platelet Hyperreactivity and Thrombosis Risk. Cell, 2016. 165(1): p. 111-24.

77. Wang, Z., Klipfell, E., Bennett, B.J., et al., Gut flora metabolism of phosphatidylcholine promotes cardiovascular disease. Nature, 2011. 472(7341): p. 57-63.

78. Koren, O., Spor, A., Felin, J., et al., Human oral, gut, and plaque microbiota in patients with atherosclerosis. Proc Natl Acad Sci U S A, 2010. 108 Suppl 1: p. 4592-8.

79. Tang, W.H., Wang, Z., Levison, B.S., et al., Intestinal microbial metabolism of phosphatidylcholine and cardiovascular risk. N Engl J Med, 2013. 368(17): p. 1575-84.

80. Koeth, R.A., Wang, Z., Levison, B.S., et al., Intestinal microbiota metabolism of L-carnitine, a nutrient in red meat, promotes atherosclerosis. Nat Med, 2013. 19(5): p. 576-85.

81. Karlsson, F.H., Fak, F., Nookaew, I., et al., Symptomatic atherosclerosis is associated with an altered gut metagenome. Nat Commun, 2012. 3: p. 1245.

82. Skagen, K., Troseid, M., Ueland, T., et al., The Carnitinebutyrobetaine-trimethylamine-N-oxide pathway and its association with cardiovascular mortality in patients with carotid atherosclerosis. Atherosclerosis, 2016. 247: p. 64-9.

83. Meir, K.S. and Leitersdorf, E., Atherosclerosis in the apolipoprotein-E-deficient mouse: a decade of progress. Arterioscler Thromb Vasc Biol, 2004. 24(6): p. 1006-14.

84. Collins, H.L., Drazul-Schrader, D., Sulpizio, A.C., et al., LCarnitine intake and high trimethylamine Noxide plasma levels correlate with low aortic lesions in ApoE(-/-) transgenic mice expressing CETP. Atherosclerosis, 2016. 244: p. 29-37.

85. Stepankova, R., Tonar, Z., Bartova, J., et al., Absence of microbiota (germ-free conditions) accelerates the atherosclerosis in ApoEdeficient mice fed standard low cholesterol diet. J Atheroscler Thromb, 2010. 17(8): p. 796-804.

86. Singh, V., Yeoh, B.S., and Vijay-Kumar, M., Gut microbiome as a novel cardiovascular therapeutic target. Curr Opin Pharmacol, 2016. 27: p. 8-12.

87. Hartstra, A.V., Bouter, K.E., Backhed, F., and Nieuwdorp, M., Insights into the role of the microbiome in obesity and type 2 diabetes. Diabetes Care, 2015. 38(1): p. 159-65.

88. Turnbaugh, P.J., Hamady, M., Yatsunenko, T., et al., A core gut microbiome in obese and lean twins. Nature, 2009. 457(7228): p. 480-4. 
89. Backhed, F., Ding, H., Wang, T., et al., The gut microbiota as an environmental factor that regulates fat storage. Proc Natl Acad Sci U S A, 2004. 101(44): p. 15718-23.

90. Backhed, F., Manchester, J.K., Semenkovich, C.F., and Gordon, J.I., Mechanisms underlying the resistance to diet-induced obesity in germ-free mice. Proc Natl Acad Sci U S A, 2007. 104(3): p. 979-84.

91. Fu, J., Bonder, M.J., Cenit, M.C., et al., The Gut Microbiome Contributes to a Substantial Proportion of the Variation in Blood Lipids. Circ Res, 2015. 117(9): p. 817-24.

92. Durgan, D.J., Ganesh, B.P., Cope, J.L., et al., Role of the Gut Microbiome in Obstructive Sleep Apnea- Induced Hypertension. Hypertension, 2016. 67(2): p. 469-74.

93. Santisteban, M.M., Kim, S., Pepine, C.J., and Raizada, M.K., Brain-Gut-Bone Marrow Axis: Implications for Hypertension and Related Therapeutics. Circ Res, 2016. 118(8): p. 1327-36.

94. Wang, Z., Roberts, A.B., Buffa, J.A., et al., Non-lethal Inhibition of Gut Microbial Trimethylamine Production for the Treatment of Atherosclerosis. Cell, 2015. 163(7): p. 1585-95.

95. Langhorne, P., Stott, D.J., Robertson, L., et al., Medical complications after stroke: a multicenter study. Stroke, 2000. 31(6): p. 1223-9.

96. Westendorp, W.F., Nederkoorn, P.J., Vermeij, J.D., Dijkgraaf, M.G., and de Beek, D., Post-stroke infection: A systematic review and meta-analysis. BMC neurology, 2011. 11: p. 110.

97. Latorre, M., Krishnareddy, S., and Freedberg, D.E., Microbiome as mediator: Do systemic infections start in the gut? World J Gastroenterol, 2015. 21(37): p. 10487-92.

98. Schuijt, T.J., Lankelma, J.M., Scicluna, B.P., et al., The gut microbiota plays a protective role in the host defence against pneumococcal pneumonia. Gut, 2016. 65(4): p. 575-83.

99. Dickson, R.P. and Cox, M.J., The premature invocation of a 'gutlung axis' may obscure the direct effects of respiratory microbiota on pneumonia susceptibility. Gut, 2016

100. Langdon, A., Crook, N., and Dantas, G., The effects of antibiotics on the microbiome throughout development and alternative approaches for therapeutic modulation. Genome Med, 2016. 8(1): p. 39.

101. Jernberg, C., Lofmark, S., Edlund, C., and Jansson, J.K., Longterm ecological impacts of antibiotic administration on the human intestinal microbiota. ISME J, 2007. 1(1): p. 56-66.

102. Meisel, A. and Smith, C.J., Prevention of stroke-associated pneumonia: where next? Lancet, 2015.

103. Springer, J., Schust, S., Peske, K., et al., Catabolic signaling and muscle wasting after acute ischemic stroke in mice: indication for a stroke-specific sarcopenia. Stroke, 2014. 45(12): p. 3675-83.

104. Scherbakov, N., Dirnagl, U., and Doehner, W., Body weight after stroke: lessons from the obesity paradox. Stroke; a journal of cerebral circulation, 2011. 42(12): p. 3646-50.

105. Turnbaugh, P.J., Backhed, F., Fulton, L., and Gordon, J.I., Dietinduced obesity is linked to marked but reversible alterations in the mouse distal gut microbiome. Cell Host Microbe, 2008. 3(4): p. 213-23.

106. Bindels, L.B., Beck, R., Schakman, O., et al., Restoring specific lactobacilli levels decreases inflammation and muscle atrophy markers in an acute leukemia mouse model. PLoS One, 2012. 7(6): p. e37971.

107. Robinson, R.G. and Jorge, R.E., Post-Stroke Depression: A Review. Am J Psychiatry, 2016. 173(3): p. 221-31.

108. Fetissov, S.O. and Dechelotte, P., The new link between gut-brain axis and neuropsychiatric disorders. Curr Opin Clin Nutr Metab Care, 2011. 14(5): p. 477-82.

109. Yarandi, S.S., Peterson, D.A., Treisman, G.J., Moran, T.H., and Pasricha, P.J., Modulatory Effects of Gut Microbiota on the Central Nervous System: How Gut Could Play a Role in
Neuropsychiatric Health and Diseases. J Neurogastroenterol Motil, 2016. 22(2): p. 201-12.

110. Forsythe, P., Sudo, N., Dinan, T., Taylor, V.H., and Bienenstock, J., Mood and gut feelings. Brain Behav Immun, 2010. 24(1): p. 916.

111. Jiang, H., Ling, Z., Zhang, Y., et al., Altered fecal microbiota composition in patients with major depressive disorder. Brain Behav Immun, 2015. 48: p. 186-94.

112. Jia, W., Li, H., Zhao, L., and Nicholson, J.K., Gut microbiota: a potential new territory for drug targeting. Nat Rev Drug Discov, 2008. 7(2): p. 123-9.

113. Ashraf, R. and Shah, N.P., Immune system stimulation by probiotic microorganisms. Crit Rev Food Sci Nutr, 2014. 54(7): p. 93856.

114. Besselink, M.G., van Santvoort, H.C., Renooij, W., et al., Intestinal barrier dysfunction in a randomized trial of a specific probiotic composition in acute pancreatitis. Ann Surg, 2009. 250(5): p. 712-9.

115. Gooszen, H.G. and Dutch Pancreatitis Study, G., The PROPATRIA trial: best practices at the time were followed. Lancet, 2010. 375(9722): p. 1249-50.

116. Dani, C., Coviello, C.C., Corsini, I.I., Arena, F., Antonelli, A., and Rossolini, G.M., Lactobacillus Sepsis and Probiotic Therapy in Newborns: Two New Cases and Literature Review. AJP Rep, 2016. 6(1): p. e25-9.

117. Oggioni, M.R., Pozzi, G., Valensin, P.E., Galieni, P., and Bigazzi, C., Recurrent septicemia in an immunocompromised patient due to probiotic strains of Bacillus subtilis. J Clin Microbiol, 1998. 36(1): p. 325-6.

118. Kochan, P., Chmielarczyk, A., Szymaniak, L., et al., Lactobacillus rhamnosus administration causes sepsis in a cardiosurgical patient-is the time right to revise probiotic safety guidelines? Clin Microbiol Infect, 2011. 17(10): p. 1589-92.

119. Eiseman, B., Silen, W., Bascom, G.S., and Kauvar, A.J., Fecal enema as an adjunct in the treatment of pseudomembranous enterocolitis. Surgery, 1958. 44(5): p. 854-9.

120. van Nood, E., Vrieze, A., Nieuwdorp, M., et al., Duodenal infusion of donor feces for recurrent Clostridium difficile. N Engl J Med, 2013. 368(5): p. 407-15.

121. Vermeire, S., Joossens, M., Verbeke, K., et al., Donor Species Richness Determines Faecal Microbiota Transplantation Success in Inflammatory Bowel Disease. J Crohns Colitis, 2016. 10(4): p. 387-94.

122. Petrof, E.O., Gloor, G.B., Vanner, S.J., et al., Stool substitute transplant therapy for the eradication of Clostridium difficile infection: 'RePOOPulating' the gut. Microbiome, 2013. 1(1): p. 3.

123. Didesch, M.M., Averill, A., and Oh-Park, M., Peripheral Neuropathy After Fecal Microbiota Transplantation for Clostridium difficile Infection: A Case Report. PM R, 2016.

124. Gregory, J.C., Buffa, J.A., Org, E., et al., Transmission of atherosclerosis susceptibility with gut microbial transplantation. J Biol Chem, 2015. 290(9): p. 5647-60.

125. Perie-FlomCenter, Bill of Health http://blogs.harvard. edu/billofhealth/2016/03/01/fda-announces-draftguidance-thatwould-limit-enforcement-discretion-for-fmt. Accessed on 29th of March 2016.

126. Belizario, J.E. and Napolitano, M., Human microbiomes and their roles in dysbiosis, common diseases, and novel therapeutic approaches. Front Microbiol, 2015. 6: p. 1050.

127. Chow, J. and Mazmanian, S.K., Getting the bugs out of the immune system: do bacterial microbiota "fix" intestinal T cell responses? Cell Host Microbe, 2009. 5(1): p. 8-12.

128. Maslowski, K.M., Vieira, A.T., Ng, A., et al. Regulation of inflammatory responses by gut microbiota and chemoattractant receptor GPR43. Nature, 2009. 461(7268): p. 1282-6. 
129. Spanogiannopoulos, P., Bess, E.N., Carmody, R.N., and Turnbaugh, P.J. The microbial pharmacists within us: a metagenomic view of xenobiotic metabolism. Nat Rev Microbiol, 2016. 14(5): p. 273-87.

130. Sousa, T., Paterson, R., Moore, V., Carlsson, A., Abrahamsson, B., and Basit, A.W. The gastrointestinal microbiota as a site for the biotransformation of drugs. Int J Pharm, 2008. 363(1-2): p. 1-25.

131. Haiser, H.J. and Turnbaugh, P.J. Is it time for a metagenomic basis of therapeutics? Science, 2012. 336(6086): p. 1253-5.

132. Wallace, B.D., Wang, H., Lane, K.T., et al. Alleviating cancer drug toxicity by inhibiting a bacterial enzyme. Science, 2010 . 330(6005): p. 831-5.

133. Vetizou, M., Pitt, J.M., Daillere, R., et al. Anticancer immunotherapy by CTLA-4 blockade relies on the gut microbiota. Science, 2015. 350(6264): p. 1079-84.
134. Sivan, A., Corrales, L., Hubert, N., et al. Commensal Bifidobacterium promotes antitumor immunity and facilitates anti-PD-L1 efficacy. Science, 2015. 350(6264): p. 1084-9.

135. Lemon, K.P., Armitage, G.C., Relman, D.A., and Fischbach, M.A. Microbiota-targeted therapies: an ecological perspective. Sci Transl Med, 2012. 4(137): p. 137rv5.

136. Kleiman, S.C., Watson, H.J., Bulik-Sullivan, E.C., et al. The intestinal microbiota in acute anorexia nervosa and during renourishment: relationship to depression, anxiety, and eating disorder psychopathology. Psychosom Med, 2015. 77(9): p. 969981.

137. Krajmalnik-Brown, R., Lozupone, C., Kang, D.W., and Adams, J.B. Gut bacteria in children with autism spectrum disorders: challenges and promise of studying how a complex community influences a complex disease. Microb Ecol Health Dis 2015. 26: p. 26914. 